\title{
The Three Strategies for Translating Source Cultural Forms under the Theory-to-strategy Analytical Framework
}

\author{
Lei Dai \\ Department of English \\ Wuhan University of Technology \\ Wuhan, China \\ dailei218@whut.edu.cn
}

\begin{abstract}
Since Venuti converted Schleiermacher's translation theory into foreignizing and domesticating, debates over the two strategies have never abated. Meanwhile, emerging translation strategies and methods are continually being named with a salient problem that it is difficult to distinguish these idiosyncratic translation strategies. This paper begins by clarifying the differences and relations between translation theories, strategies and methods, emphasizing that strategies be converted from theories. Based on the theory-to-strategy analytical framework, the interpretive theory and the dynamic formal equivalence theory are converted into clarifying, the third strategy for source cultural forms. Applications of the three strategies are illustrated with examples.
\end{abstract}

Keywords-source cultural forms; theory-to-strategy analytical framework; clarifying

\section{SCHLEIERMACHER'S TRANSLATION THEORY AND VENUTI'S FOREIGNIZING AND DOMESTICATING TRANSLATION STRATEGIES}

In 1813, Schleiermacher proposed one of the kernel theories in Translation Studies that deals with the cultural forms of the source text: "you can either push the reader towards the writer or push the writer towards the reader" [1]. In the early 1990s, Venuti substantiated this metaphoric theory into two polarized translation strategies: foreignizing and domesticating. "Foreignizing means that the translator tries maximally to retain the source cultural forms in the translated text while domesticating means that the source cultural forms are replaced with their counterparts or equivalents in the target language" [2]. As a matter of fact, the two strategies echo the then emerging cultural turn theory which emphasizes that translation be deemed as the cultural product of the target language and that translation strategies are inevitably influenced by the very sociocultural context where the text is rendered. The centerpiece of the theory reflects the two distinct ways in processing the source cultural forms: either maximally retaining it or replacing it with the target cultural equivalents. Therefore, there is certain homogeneity or unanimity between Schleiermacher's theory, Venuti's foreignizing and domesticating and the cultural turn theory. Since the inception

This research was financially supported by the Fundamental Research Funds for the Central Universities, PRC on the project On the English Translation of the Core Political Concepts of China: A Parallel Corpus Approach (program code: 2019VI004). of foreignizing and domesticating, there has been a prolonged controversy over them within the circle of translation. Despite that, a consensus has been basically reached that there is nothing but those two strategies.

With the rapid development of Translation Studies as an academic discipline, new and emerging strategies, skills and methods of translation have been mushrooming, seeming to transcend the stereotyped dualism of foreignizing and domesticating. Their main drawback is that it is idiosyncratic and subjective of the researchers to haphazardly name their manifold strategies which are simply explained with the random examples selected from translated texts. But it remains unsettled how to distinguish the various strategies proposed by different researchers, how to verify them and particularly how to classify those homogeneous strategies that bears different names. And this prompts us to ponder about the differences between such concepts as translation theories, translation strategies and translation methods as well as the connections between these new strategies and the binary foreignizing and domesticating.

\section{DiffERENCES AND CONNECTIONS BETWEEN TRANSLATION THEORIES, STRATEGIES AND METHODS}

After investigating the discussions on this issue among scholars both home and abroad, this paper presents that translation theory, as a description of translation studies, attempts to generalize the principles and rules of the various translation phenomena and activities and to answer what is translation, how the translator works and many other fundamental questions. As part of the evolution of translation theories, translation strategies illustrate how a translation purpose can be achieved given the manifold contextual constraints. Translation methods or skills are aimed to tackle specific translation problems on the textual levels. With regard to the relations between the three, Boase-Beier once pointed out that "certain translation theories cannot be applied to translation practice until after they are in the first place converted into translation strategies” [3]. So it is argued in this paper that the relationship between the three concepts is: translation theory can be converted into translation strategy which can be further converted into translation strategies, as indicated in figure 1 . 
It is therefore essential to firstly distinguish whether it is in nature a strategy or a method before researching a translation strategy. Secondly, researchers are supposed to be aware that there is only a limited number of strategies which can only be converted from theories. As aforementioned, Schleiermacher's classic metaphor serves as the feeding theory for Venuti's foreignizing and domesticating translation strategies. Likewise, many a method has derived from the two strategies. For example, the name of mascots for the 2008 Beijing Summer Olympics was translated according to its Chinese pronunciation as Fuwa (literally happy kids who can bring in good luck). Seen from strategies, the translation adopted foreignizing, since the specific cultural characteristics are preserved while in the perspective of methods, Fuwa used transliteration. Concerning the inter-connectivity between Schleiermacher's theory and Venuti's binary strategies, by using foreignizing, the translator pushes the reader towards the writer by maximally retaining source cultural forms in the target text while s/he pushes the writer towards the reader by replacing the source cultural forms with those equivalents in the target language. Inspired by this classic conversion, the paper proposes the theory-to-strategy analytical framework of translation, namely converting translation theories into corresponding translation strategies, so as to confirm if there are only two translation strategies to deal with source cultural forms.

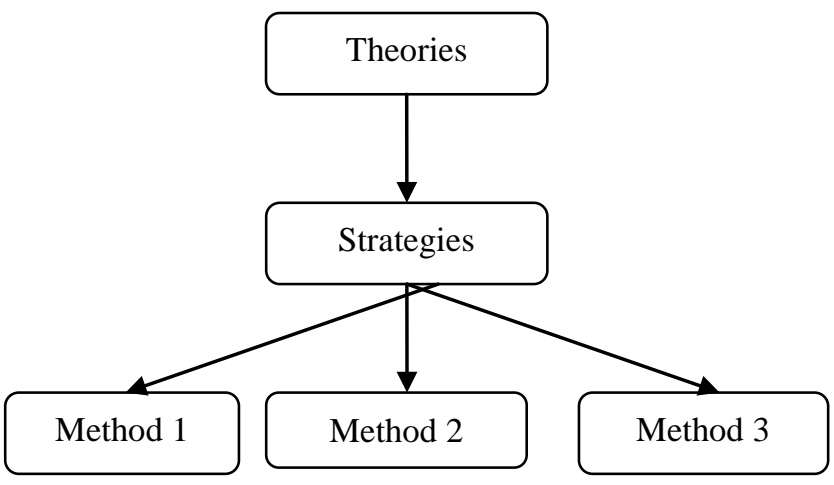

Fig. 1. Hierarchy of Translation Theories, Strategies and Methods

\section{THE THEORY-TO-STRATEGY ANALYTICAL FRAMEWORK AND THE THREE TRANSLATION STRATEGIES FOR SOURCE CULTURAL FORMS}

\section{A. The Clarifying Strategy: Converted from Core Theories of Translation and Interpreting}

Thanks to the theory-to-strategy analytical framework, researchers are able to maximally minimize their subjectivity and idiosyncrasy in naming translation strategies, because these strategies will have theories behind them, or in other words, they are converted from theories. Conversely, when analyzing texts, researchers can explain their selection of certain translation strategies with specific contextual factors and translation theories.

Regarding the ways to deal with source cultural forms, both interpreting and translation theories can be put into the right perspective. Seen from the interpretive theory of translation in interpreting studies, "what counts most for an interpreter is the meaning instead of the linguistic forms of the source text" [4]. Just as language is the carrier of culture, the source cultural forms are per se a kind of linguistic form. In other words, meaning is prioritized over form in interpreting, as posited by the interpreting theory of translation. Likewise, Nida's dynamic equivalence theory on (written) translation refutes the absolutism of translatability, stressing that "meaning be prioritized over form when conflicts arise between the functional purpose(s) of the source text and its aesthetic / cultural / artistic forms" [5]. Actually, both theories have touched upon the notion of "cultural gaps" [6] which emerge during the linguistic and cultural conversion. These gaps directly result into intranslatability; to compensate for this, the meaning of the source text is conveyed as faithfully as it can possibly be at the expense of the linguistic / cultural forms which are more often than not clarified in the target text.

Given the two influential theories respectively in translation and interpreting studies, clarifying, the third strategy for translating source cultural forms, is hereby proposed. Thanks to it, the source cultural forms are neither retained nor replaced but removed, with only the most basic concrete meaning delivered. With this strategy, both the reader and the writer jointly approach each other, shortening together their distance, as shown in Fig. 2.

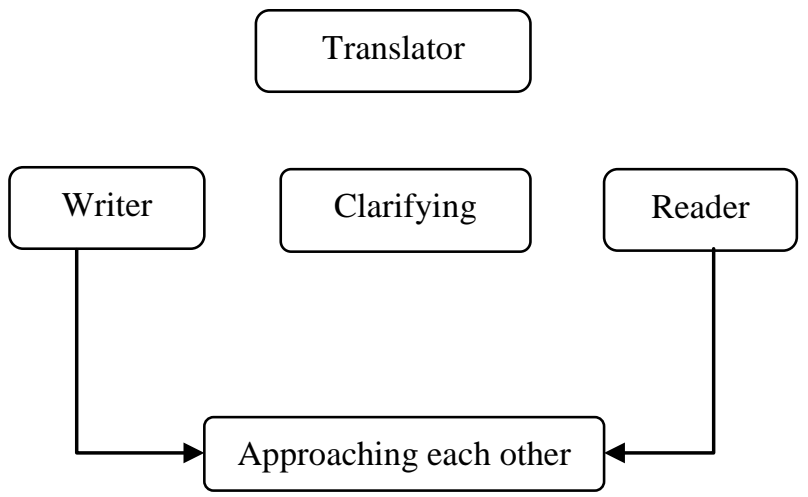

Fig. 2. The Clarifying Strategy

\section{B. Application of Foreignizing, Domesticating and Clarifying}

Confusion arises among readers with the source cultural forms that are considered as cultural gaps because they are directly transplanted from the source to the target text using foreiginizing. By contrast, source cultural forms are replaced with the target text counterparts through domesticating to make the translation comprehensible to readers. Therefore, it seems that there is a dilemma between the preservation of the source cultural forms and the easiness to comprehend the target text. We have to ponder about the choice of strategies in line with the specific contexts. Taking a well-known quote of Mencius, a renowned sage in ancient China, as an example, we can derive three versions from the three strategies.

- Source text: You can't have both fish and bear's paw concurrently. (meanwhile the foreignized version)

- Domesticated version: You can’t have your cake and eat it too. 
- Clarified version: In order to have something, you may have to sacrifice something else.

Obviously, the foreignized version has transferred the original symbols of fish and bear's paw directly from Chinese into English. Native English respondents in this study told the researcher that they could not figure out its true meaning, which results from the reckless pushing of readers towards the writer or in other words forcing the unique Chinese cultural expressions into the minds of the foreign readers. "Overemphasizing the exotic source culture in cross-cultural communication is equivalent to forcing the unpalatable traditional Chinese herbs into patients' throat” [7]. Despite that, foreignizing has its time, particularly when there is a need to introduce its literal meaning and connotation to foreign receivers, as described in the foreignized version. However, under most circumstances, domesticating or clarifying is needed to facilitate understanding. Clearly, the cake metaphor in the domesticated version has replaced the fish-and-bear one, pushing the writer to the reader by modifying the source text for better comprehension. Similarly, the clarified version is primarily to transfer meaning, but distinct from the replacement mindset of domesticating, it conveys the kernel message in a concise and straightforward manner. In effect, readers and the writer are drawn near to each other thanks to it, with the source cultural forms neither replaced nor retained.

Yet as Verschueren posited "there are numerous linguistic choices and we have many different ways to express the same message" [8]. Therefore, although using the strategy, we can have different versions. Still taking the above quote as an example, we can also say "you can't have your water and drink it too". Using clarifying, we can alternatively translate it as "You can't always get everything you want". Besides, the three strategies are not necessarily used alone. In other words, at times two strategies can be used in collocation. For example, as for the Chinese Government's anti-corruption slogan "to battle tigers and flies" (literal meaning), there have been various translated versions. Based on the three strategies proposed in this paper, we can classify them into three categories.

- Version 1: To battle tigers and flies. Tigers and flies refer to high-ranking and low-ranking corrupt officials respectively. (foreignizing plus clarifying)

- Version 2: To bring corrupt officials of all levels to justice. (clarifying)

- Version 3: To bring all corrupt officials to justice, be they big shots or small potatoes. (clarifying plus domesticating)

As investigated by the researcher of the paper, when firstly proposed, the slogan was translated as version 1 by BBC, namely foreignizing first before clarifying the connotations of tigers and flies, therefore impressing the western readership with not only its literal references but also its implicature. Version 2 is more frequently found in the annual Chinese Premier's Press Conference held in Beijing and the strategy is expected to enable the foreign journalists to quickly make sense of the metaphor as well as delivering the message accurately. The third version was proposed by Prof. Gong Naixu of Beijing Service Bureau for Diplomatic Missions.
Similar to the first half of version 2, this one substantiates the content of the slogan while its second half is domesticating. In Oxford Advanced English-English Dictionary (7th Edition), "big shots” means "an important or influential person” while "small potatoes" means "something insignificant or unimportant". Stylistically, "big shots and small potatoes" correspond to the vivid cultural metaphor of "tigers and flies".

Therefore, the example illustrates that the translated version can be different given different translation strategies despite the generally same message of the source text. Likewise, different translation effects can be achieved in line with the specific translation purposes under ad-hoc contexts using the three strategies. And all these analyses have again confirmed Nida's dynamic equivalence theory in that equivalence of meaning is affected by the very context where a translation activity takes place. Besides, translatability is not fixed exclusively on the textual level but posteriori and subject to changes of any subtle contextual factor, including time, venue, the target readership, and the purpose for translation and so on. The translation of a same novel for college students majoring in British Literature would definitely be distinct from that for primary school children.

\section{CONCLUSION}

Based on further clarifying the differences and connections between translation theories, strategies and methods, this paper has pointed out the limitations of previous studies on translation strategies, namely, researchers had subjectively and haphazardly proposed various idiosyncratic strategies that were hard to tell one from another. The theory-to-strategy analytical framework proposed in this research, however, elaborated on how foreignizing and domesticating are converted from Schleiermacher's translation theory, and converted into clarifying, the third translation strategy for source cultural forms from the interpretive theory of translation and the dynamic equivalence theory. Clarifying means that the translator focuses only on the delivery of the most basic meaning of the source text rather than hesitate over whether to retain or replace the source cultural forms. Through example illustrations, the paper has compared and expounded the applications of the three strategies to the processing of source cultural forms. The translation theory-to-strategy analytical framework is expected to be applied to future studies on translation strategies.

\section{REFERENCES}

[1] F. Schleiermacher, "On the different methods of translating," in The Translation Studies Reader, L. Venuti, Eds. London: Routledge, 2004, pp. 43-63.

[2] L. Venuti, The Translator's Invisibility: A History of Translation. New York: Routledge, 1995.

[3] J. Boase-Beier, A Critical Introduction to Translation Studies. London: Continuum, 2011.

[4] J. L. Zhang, On the theoretical achievements of the Paris Interpretive School, Chinese Science \& Technology Translators Journal, vol. 4, pp. 16-19, 2009.

[5] E. A. Nida, Toward a Science of Translating. Leiden: E. J. Brill, 1964. 
[6] W. L. Ma, Analyzing Chinglish in Chinese media, Journalism \& Communication Review, vol. 4, pp. 464-467, 2009.
[7] Y. F. Sun, Translation and cross-cultural strategies, Chinese Translators Journal, vol. 1, pp. 16-23, 2012.

[8] J. Verschueren, Understanding Pragmatics. London: Arnold, 1999. 